\title{
Review of: "Postnatal development of centrifugal inputs to the olfactory bulb"
}

\author{
Harvey Sarnat ${ }^{1}$ \\ 1 Alberta Health Services
}

Potential competing interests: The author(s) declared that no potential competing interests exist.

This manuscript describes the feedback reciprocal inputs to the olfactory bulb from the secondary (anterior olfactory nucleus) and tertiary (entorhinal cortex/piriform lobe; amygdala; hippocampus) targets of primary olfactory bulb mitral cells. This is a study in early postnatal mice and perhaps that detail should be added to the title. The number of mice studied is not stated in either the Materials and Methods section or in the Abstract, but should be clarified in both places.

The data presented support the authors' conclusions that there is an increase of these centrifugal projections in the immediate postnatal period, important and novel data not previously well documented.

In their Discussion, the authors might consider addressing the deep core of the olfactory bulb and extending into the olfactory tract, comprised of axonless granular GABAergic neurons with axo-dendritic synapses which, together with the periglomerular neurons that also form dendrodendritic synapses, form an intrinsic olfactory thalamic equivalent; the olfactory is the only special sensory system to not project primarily to the thalamus (Sarnat HB, Yu W. Maturation and dysgenesis of the human olfactory bulb. Brain Pathol 2016;26:301-318). How does this “intrinsic olfactory thalamus" interact with the centrifugal connections that the authors describe? The granule cell layer of the olfactory bulb is not yet mature at birth in humans, in terms of expression of mature neuronal proteins and synaptogenesis (see Sarnat, Yu reference above). Granular neurons are entirely intrinsic with no projections outside the olfactory bulb, not surprising since they lack axons.

Another useful addition to the Discussion might be a brief comparison of the murine olfactory system with the human, beginning with stating the gestational age in humans that corresponds to the day of birth of the mouse. Are there data on the development of synapses in the maturing olfactory bulb of the mouse to compare 
with the human study noted above? Myelination?

The manuscript is well written with good English grammar. References cited are appropriate in number and selection. The figures are of good quality and help document findings described in the text. 